\title{
Sparse Representation Denoising for Radar High Resolution Range Profiling
}

\author{
Min Li, ${ }^{1}$ Gongjian Zhou, ${ }^{2}$ Bin Zhao, ${ }^{3}$ and Taifan Quan ${ }^{4}$ \\ ${ }^{1}$ Harbin Institute of Technology, Room 807, Building 20, 92 West Dazhi Street, Harbin 150001, China \\ ${ }^{2}$ Harbin Institute of Technology, Room 705, Building 20, 92 West Dazhi Street, Harbin 150001, China \\ ${ }^{3}$ Harbin Institute of Technology, Room 802, Building 20, 92 West Dazhi Street, Harbin 150001, China \\ ${ }^{4}$ Harbin Institute of Technology, Room 708, Building 20, 92 West Dazhi Street, Harbin 150001, China
}

Correspondence should be addressed to Taifan Quan; quantf@126.com

Received 15 November 2013; Accepted 11 February 2014; Published 17 March 2014

Academic Editor: Margaret Cheney

Copyright (C) 2014 Min Li et al. This is an open access article distributed under the Creative Commons Attribution License, which permits unrestricted use, distribution, and reproduction in any medium, provided the original work is properly cited.

\begin{abstract}
Radar high resolution range profile has attracted considerable attention in radar automatic target recognition. In practice, radar return is usually contaminated by noise, which results in profile distortion and recognition performance degradation. To deal with this problem, in this paper, a novel denoising method based on sparse representation is proposed to remove the Gaussian white additive noise. The return is sparsely described in the Fourier redundant dictionary and the denoising problem is described as a sparse representation model. Noise level of the return, which is crucial to the denoising performance but often unknown, is estimated by performing subspace method on the sliding subsequence correlation matrix. Sliding window process enables noise level estimation using only one observation sequence, not only guaranteeing estimation efficiency but also avoiding the influence of profile time-shift sensitivity. Experimental results show that the proposed method can effectively improve the signal-to-noise ratio of the return, leading to a high-quality profile.
\end{abstract}

\section{Introduction}

Radar high resolution range profile (HRRP) contains rich information for target feature analysis, such as target structure and scatterer distribution, and has received considerable attention in radar automatic target recognition (ATR) in recent years [1-7]. HRRP feature selection and extraction have been investigated in $[1,2]$. Parametric statistical models have been applied to HRRP ATR [3-5]. High order spectra and complex HRRP features have been studied for target classification [6,7]. In these works, high signal-to-noise ratio (SNR) is a necessary condition. However the target return is usually contaminated by noise in practice. This results in HRRP signature distortion and recognition performance degradation [8].

Some methods have been proposed to handle this problem in target recognition stage. The noise-robust bispectrum features have been extracted for target recognition [9]. Modifying the statistical model parameters according to the noise level of test samples is developed in [10], but this method suffers from a high computational burden.

Noise-robust imaging is another effective approach. In [11], higher order statistics is presented for imaging at low SNR, which however has some information loss. Recently, noise-robust compressive sensing (CS) imaging is developed $[12,13]$. In these methods, a key problem is how to estimate the noise level, which impacts the quality of image because of the noise sensitivity of CS. In [12] the noise level is estimated by computing the energy of the noise cells, which are selected by an energy-based threshold. The estimation accuracy of this method relies on threshold selection and number of noise cells. In [13], the noise variance is estimated by employing the median value of finest scale wavelet coefficients. Usually, HRRP is obtained from one observation sequence, in which the number of samples is limited. In this scenario, these methods cannot guarantee the estimation accuracy of noise level. 
Another natural choice is to remove noise in radar return. Wavelet shrinkage was shown to be effective when SNR is beyond a certain threshold $[14,15]$. However its performance degrades largely in the presence of severe noise contamination. Combined bispectrum-filtering is used to suppress noise in HRRP to improve recognition performance [16, 17], but this method requires a large number of observations to average bispectrum estimates. In addition, for some common profiling processes, for example, dechirping for wideband signal, the dechirped output of target return contains multiple frequency components, which change with observations due to HRRP time-shift sensitivity [18]. In this case, the requirement of a large number of observations cannot be satisfied.

Sparse representation (SR) theory shows that, in sparse and redundant dictionary, signal energy concentrates on minority atoms, whereas noise energy spreads over almost all the atoms, and the signal can be stably recovered from noisy measurements [19-21]. Based on this, in this paper, we propose a sparse representation denoising method to improve the SNR of noisy return for profiling. A Fourier redundant dictionary is established to sparsely describe the return and the denoising problem is described as a sparse representation model. Noise reduced return is recovered by solving the sparse representation problem. The noise level, which is a key parameter for denoising by sparse representation, is estimated by preforming subspace method on the subsequence correlation matrix. In this way, the noise level can be obtained only using one observation sequence, not only guaranteeing the efficiency but also avoiding the influence of HRRP timeshift sensitivity. Simulated and real data experiments are provided to verify the effectiveness of the proposed denoising method.

The rest of this paper is organized as follows. The basic theory of radar high resolution range profiling is introduced in Section 2. Radar return denoising by sparse representation is discussed in Section 3. The simulated and real data experiments are provided to verify the denoising performance in Section 4, followed by conclusions in Section 5 .

\section{Radar High Resolution Range Profiling}

Usually, HRRP is obtained by wideband signal, such as linear frequency modulation (LFM) signal and steppedfrequency (SF) signal. In this paper, we take the LFM signal as an example to introduce the basic theory of radar high resolution range profiling by dechirping process [22, 23]. The transmitted wideband LFM signal can be represented as

$$
s(t)=\operatorname{rect}\left(\frac{t}{T_{p}}\right) \exp \left\{j 2 \pi\left(f_{c} t+\frac{1}{2} \gamma t^{2}\right)\right\},
$$

where

$$
\operatorname{rect}(u)= \begin{cases}1 & |u| \leq \frac{1}{2} \\ 0 & |u|>\frac{1}{2}\end{cases}
$$

denotes complex signal envelope, $f_{c}$ denotes carrier frequency, $T_{p}$ is the pulse width, and $\gamma$ is the frequency modulation slope.

Given reference range $R_{\text {ref }}$, the dechirping reference signal can be represented as

$$
\begin{aligned}
s_{\text {ref }}(t)= & \operatorname{rect}\left(\frac{t-2 R_{\text {ref }} / c}{T_{\text {ref }}}\right) \\
& \times \exp \left\{j 2 \pi\left[f_{c}\left(t-\frac{2 R_{\text {ref }}}{c}\right)+\frac{1}{2} \gamma\left(t-\frac{2 R_{\text {ref }}}{c}\right)^{2}\right]\right\},
\end{aligned}
$$

where $T_{\text {ref }}$ is the pulse width of reference signal, usually larger than $T_{p}$. Assuming a scatterer locating at range $R_{t}$, the return from the scatterer is

$$
\begin{aligned}
s_{r}(t)= & A \cdot \operatorname{rect}\left(\frac{t-2 R_{t} / c}{T_{p}}\right) \\
& \times \exp \left\{j 2 \pi\left[f_{c}\left(t-\frac{2 R_{t}}{c}\right)+\frac{1}{2} \gamma\left(t-\frac{2 R_{t}}{c}\right)^{2}\right]\right\},
\end{aligned}
$$

where $A$ denotes the return amplitude. The dechirping output is

$$
s_{i f}(t)=s_{r}(t) \cdot s_{\mathrm{ref}}^{*}(t),
$$

where $*$ stands for complex conjugate. Let $R_{\Delta}=R_{t}-R_{\text {ref }}$, and after some manipulations, (5) can be rewritten as

$$
\begin{aligned}
s_{i f}(t) & A \cdot \operatorname{rect}\left(\frac{t-2 R_{t} / c}{T_{p}}\right) \\
& \times \exp \left\{-j \frac{4 \pi}{c} \gamma\left(t-\frac{2 R_{\mathrm{ref}}}{c}\right) R_{\Delta}-j \frac{4 \pi}{c} f_{c} R_{\Delta}+j \frac{4 \pi \gamma}{c^{2}} R_{\Delta}^{2}\right\} \\
= & A \cdot \operatorname{rect}\left(\frac{t-2 R_{t} / c}{T_{p}}\right) \\
& \times \exp \left(-j \frac{4 \pi}{c} \gamma R_{\Delta} t+j \frac{8 \pi R_{\mathrm{ref}}}{c^{2}} \gamma R_{\Delta}-j \frac{4 \pi}{c} f_{c} R_{\Delta}-j \frac{4 \pi}{c} f_{c} R_{\Delta}\right) .
\end{aligned}
$$

The later three phase terms in (6) are constants, with no contribution to HRRP. Denoting the sum of the later three phase terms by $\varphi,(6)$ can be rewritten as

$$
\begin{aligned}
s_{i f}(t) & =A \cdot \operatorname{rect}\left(\frac{t-2 R_{t} / c}{T_{p}}\right) \exp \left(-j \frac{4 \pi}{c} \gamma R_{\Delta} t+j \varphi\right) \\
& =A \cdot \operatorname{rect}\left(\frac{t-2 R_{t} / c}{T_{p}}\right) \exp \left(-j 2 \pi f_{d} t+j \varphi\right),
\end{aligned}
$$

where $f_{d}=2 \gamma R_{\Delta} / c$. Formula (7) shows that the dechirped output of the return from one scatterer is a complex sinusoidal signal with a frequency proportional to its relative range. 


\section{Denoising by Sparse Representation}

In this section, we start the presentation of the proposed denoising method by first discussing how redundant dictionary is established for sparsely describing the dechirped return. Once the sparse representation model is established, the noise level is estimated by subspace method, followed with the reconstruction of the denoised signal by solving sparse representation.

3.1. Dechirped Return Sparse Representation. In noisy circumstance, assume a target contains $K$ scatterers locating at different ranges and there are $M$ sampling points in a single pulse. According to (7), the time domain sampling sequence of the dechirping output pulse can be represented as

$$
\begin{aligned}
y(m)= & s(m)+n(m) \\
= & \sum_{k=1}^{K} A_{k} \cdot \exp \left(-j 2 \pi f_{k} m+j \varphi_{k}\right)+n(m), \\
& m=0,1, \ldots, M-1,
\end{aligned}
$$

where $A_{k}, f_{k}$, and $\varphi_{k}$ are the amplitude, relative frequency normalized by sampling rate, and constant phase of the return from the $k$ th scatterer, respectively. $s(m)$ and $n(m)$ denote signal and the Gaussian white noise sampling sequences, respectively. Let $\mathbf{y}=[y(0), y(1), \ldots, y(M-1)]^{T}, \mathbf{s}=$ $[s(0), s(1), \ldots, s(M-1)]^{T}$, and $\mathbf{n}=[n(0), n(1), \ldots, n(M-1)]^{T}$; then (8) can be rewritten as

$$
\mathbf{y}=\mathbf{s}+\mathbf{n}=\sum_{k=1}^{K} u_{k} \cdot \mathbf{v}_{k}+\mathbf{n},
$$

where $u_{k}=A_{k} \exp \left(j \varphi_{k}\right)$ and $\mathbf{v}_{k}=\left[1, \exp \left(-j 2 \pi f_{k}\right), \ldots\right.$, $\left.\exp \left\{-j 2 \pi f_{k}(M-1)\right\}\right]^{T}$. Formula (9) indicates that the dechirped output sequence contains multiple complex sinusoidal components superposed with noise. Usually, the number of the main scatterers of a target is much less than that of the range cells in the pulse. Thus $\mathbf{s}$ is sparse in frequency domain and can be sparsely represented by the complex Fourier redundant dictionary, which is constructed as

$$
\mathbf{A}=\left\{\phi_{1}, \phi_{2}, \ldots, \phi_{N}\right\} \in \mathbb{C}^{M \times N},
$$

where

$$
\boldsymbol{\phi}_{i}=\exp \left\{-j 2 \pi \cdot \mathbf{f}_{N}(i) \cdot \mathbf{m}\right\}, \quad i=1, \ldots, N,
$$

$\mathbf{m}=[0,1, \ldots, M-1]^{T}$, and $N>M$. In $(11), \mathbf{f}_{N}=[0,1 / N$, $\ldots,(N-1) / N]$ is the normalized frequency. Then (9) can be represented as

$$
\mathbf{y}=\mathbf{A x}+\mathbf{n},
$$

where $\mathbf{x}$ is a sparse vector, composed of the decomposition coefficients of signal $\mathbf{s}$ in $\mathbf{A}$. Sparse representation theory shows that if $\mathbf{x}$ satisfies $\|\mathbf{x}\|_{0}<(1 / 2) \operatorname{spark}(\mathbf{A}), \mathbf{x}$ can be stably solved by the following $l_{0}$ optimization problem [21]:

$$
\widehat{\mathbf{x}}=\arg \min \|\mathbf{x}\|_{0} \quad \text { s.t. }\|\mathbf{y}-\mathbf{A x}\|_{2} \leq \delta,
$$

where $\|\cdot\|_{0}$ stands for the $l_{0}$ norm of a vector (i.e., the number of its nonzero elements), $\operatorname{spark}(\mathbf{A})$ denotes the minimum number of linearly dependent columns of $\mathbf{A}$, and $\delta$ is the noise level. It is hard to solve (13). Approximated solution can be acquired by greedy algorithms, for example, orthogonal matching pursuit (OMP) [24]. In this paper, OMP is utilized to solve (13) because of its simplicity and efficiency. When obtaining $\widehat{\mathbf{x}}$ from (13), the denoised signal can be acquired by $\widehat{\mathbf{s}}=\mathbf{A} \widehat{\mathbf{x}}$

3.2. Estimation of Noise Level. In solving (13) by OMP, the noise level $\delta$ is a crucial parameter, which is the iteration terminal condition of OMP. However, in most cases, $\delta$ is unknown and thus needs to be estimated. An underestimated $\delta$ will introduce extra noise components in the recovered signal, and the overestimated one will cause some scatterer information loss. Hence precise estimation of noise level is necessary.

Subspace method combined with minimum description length (MDL) criterion is an effective method for detecting the number of signals and estimating the signal-tointerference ratio (SIR) [25-27]. To guarantee the estimation performance, a number of observations are needed to evaluate sample covariance matrix; meanwhile the signal frequencies should not change during these observations. However, the frequency components in the dechirped radar return vary significantly with observations due to the HRRP time-shift sensitivity [18]. As a result, it is hard to incorporate multiple consecutive observations to estimate noise power as [27]. On the other hand, collecting multiple observations to estimate noise power impairs the efficiency. In this paper, the noise level is estimated by subspace method with only one observation sequence rather than multiple observations.

In general, the number of sampling points within a single pulse is much more than that of the target scatterers and the dechirped return can be assumed to be a stationary signal. Sliding window process can be adopted to obtain subsequences, which are used to construct subsequence correlation matrix. According to (8), assuming one observation sequence of the dechirped signal is $y(m), m=0,1, \ldots, M-1$, subsequences are constructed as

$$
\begin{array}{r}
\mathbf{y}_{i}=[y(i), y(i+1), \ldots, y(i+L-1)]^{T}=\mathbf{s}_{i}+\mathbf{n}_{i}=\mathbf{A} \boldsymbol{\alpha}_{i}+\mathbf{n}_{i}, \\
i=0,1, \ldots, M-L,
\end{array}
$$

where $\mathbf{A}=\left[\mathbf{A}_{1}, \mathbf{A}_{2}, \ldots, \mathbf{A}_{K}\right]$ denotes subsequence representation basis matrix, $\mathbf{A}_{k}=\left[1, \exp \left(-j 2 \pi f_{k}\right), \exp \left(-j 2 \pi 2 f_{k}\right), \ldots\right.$, $\left.\exp \left(-j 2 \pi(L-1) f_{k}\right)\right]^{T}, k=1,2, \ldots, K$ is the $k$ th basis of matrix $\mathbf{A}$, and $\boldsymbol{\alpha}_{i}=\left[A_{1} \exp \left(-j 2 \pi i f_{1}+j \varphi_{1}\right), A_{2} \exp \left(-j 2 \pi i f_{2}+j \varphi_{2}\right)\right.$, $\left.\ldots, A_{K} \exp \left(-j 2 \pi i f_{K}+j \varphi_{K}\right)\right]^{T}$ is the representation coefficient vector of $\mathbf{y}_{i}$. The subsequence correlation matrix is given by

$$
\widehat{\mathbf{R}}=\frac{1}{M-L+1} \sum_{i=0}^{M-L} \mathbf{y}_{i} \mathbf{y}_{i}^{H},
$$

where $H$ denotes conjugate transpose. Let $L>K$. The expectation of $\widehat{\mathbf{R}}$ is

$$
\widehat{\mathbf{R}}_{E}=\widehat{\mathbf{R}}_{S}+\widehat{\mathbf{R}}_{n}=\widehat{\mathbf{R}}_{S}+\sigma^{2} \mathbf{I},
$$


where $\widehat{\mathbf{R}}_{n}=\sigma^{2}$ I denotes the noise correlation matrix, $\sigma^{2}$ is a scalar constant denoting noise power, $\mathbf{I}$ is the identify matrix, and

$$
\begin{aligned}
\widehat{\mathbf{R}}_{S} & =\frac{1}{M-L+1} \sum_{i=0}^{M-L} \mathbf{s}_{i} \mathbf{s}_{i}^{H} \\
& =\frac{1}{M-L+1} \sum_{i=0}^{M-L} \mathbf{A} \boldsymbol{\alpha}_{i} \boldsymbol{\alpha}_{i}^{H} \mathbf{A}^{H}=\frac{1}{M-L+1} \mathbf{A} \mathbf{P} \mathbf{A}^{H}
\end{aligned}
$$

with $\mathbf{P}=\sum_{i=0}^{M-L} \boldsymbol{\alpha}_{i} \boldsymbol{\alpha}_{i}^{H}=\boldsymbol{\beta} \boldsymbol{\beta}^{H}, \boldsymbol{\beta}=\left[\boldsymbol{\alpha}_{0}, \boldsymbol{\alpha}_{1}, \ldots, \boldsymbol{\alpha}_{M-L}\right] . \widehat{\mathbf{R}}_{S}$ stands for the signal correlation matrix. Let $M-L+1>L>K$. The matrix $\boldsymbol{\beta}$ is row linearly independent; namely, the rank of $\boldsymbol{\beta}$ is $K$. It follows that the rank of $\widehat{\mathbf{R}}_{S}$ is $K$; equivalently, the $L-K$ smallest eigenvalues of $\widehat{\mathbf{R}}_{S}$ are all equal to zero. Thus $\widehat{\mathbf{R}}_{S}$ can be diagonalized by

$$
\mathbf{U}^{H} \widehat{\mathbf{R}}_{S} \mathbf{U}=\Lambda_{S},
$$

where $\mathbf{U}$ is an orthogonal matrix and $\boldsymbol{\Lambda}_{S}=\operatorname{diag}\left[\lambda_{1}, \lambda_{2}, \ldots\right.$, $\left.\lambda_{K}, 0, \ldots, 0\right], \lambda_{1} \geq \lambda_{2} \geq \cdots \geq \lambda_{K}$. In the same way, $\widehat{\mathbf{R}}_{E}$ can be diagonalized as

$$
\mathbf{U}^{H} \widehat{\mathbf{R}}_{E} \mathbf{U}=\boldsymbol{\Lambda}_{Y}=\boldsymbol{\Lambda}_{S}+\sigma^{2} \mathbf{I},
$$

where $\Lambda_{Y}=\operatorname{diag}\left[\lambda_{1}+\sigma^{2}, \lambda_{2}+\sigma^{2}, \ldots, \lambda_{K}+\sigma^{2}, \sigma^{2}, \ldots, \sigma^{2}\right]$ is the eigenvalue matrix of $\widehat{\mathbf{R}}_{E}$. It seems that the noise power can be estimated by observing the rest of the smallest $L-K$ eigenvalues of $\widehat{\mathbf{R}}_{E}$. However, in practice, because of the finite length of sampling sequence, the smallest $L-K$ eigenvalues are usually not equal to each other. In this case, we can utilize minimum description length (MDL) criterion to detect the noise components and the noise power can be estimated by averaging the rest of the smallest $L-K$ eigenvalues [27].

According to the above analysis, for a $M \times 1$ noisy sequence $\mathbf{y}$, the procedure of estimating noise level $\delta$ can be summarized as follows.

(1) Estimate the subsequence correlation matrix by (15).

(2) Perform eigenvector decomposition of the correlation matrix as $\widehat{\mathbf{R}}=\mathbf{U} \boldsymbol{\Lambda} \mathbf{U}^{H}$, where

$\Lambda=\operatorname{diag}\left(l_{i}\right), \quad i=1,2, \ldots, L, l_{1} \geq l_{2} \geq \cdots \geq l_{L}$.

(3) For $p=0,1, \ldots, L-1$, compute the MDL function:

$$
\begin{aligned}
\operatorname{MDL}(p) & \\
= & -(M-L+1)(L-p) \log \left(\frac{\prod_{i=p+1}^{L} l_{i}^{1 /(L-p)}}{(1 /(L-p)) \sum_{i=p+1}^{L} l_{i}}\right) \\
& +0.5 p(2 L-p) \log (M-L+1) .
\end{aligned}
$$

(4) Find the index $\widehat{K}$ that minimizes the MDL function; that is,

$$
\widehat{K}=\underset{p=0,1, \ldots, L-1}{\arg \min } \operatorname{MDL}(p)
$$

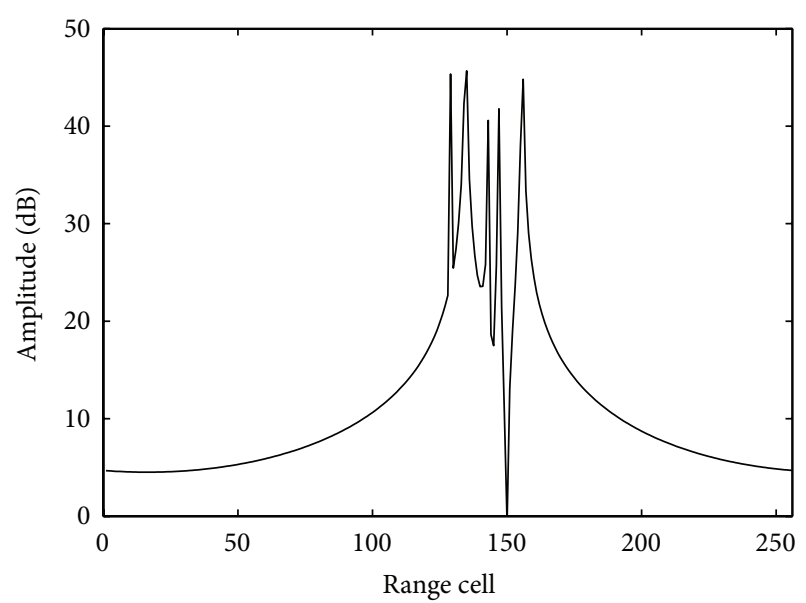

FIgURE 1: Noiseless HRRP of simulated target.

(5) Estimate the noise power by averaging the rest of the smallest $L-\widehat{K}$ eigenvalues as

$$
\widehat{\sigma}^{2}=\frac{1}{L-\widehat{K}} \sum_{i=\widehat{K}+1}^{L} l_{i} .
$$

(6) Calculate the noise level as

$$
\widehat{\delta}=\sqrt{M \widehat{\sigma}^{2}} .
$$

In the above procedure, the noise level is estimated using only one-observation data and thus this denoising method can be used to deal with observations at any unknown SNR. We refer to this denoising method as sparse representation denoising with noise level estimation by subsequence (SRDN-NS).

SRDN-NS contains two stages: noise level estimation and the sparse-representation solving. Hence its computational complexity can be divided into parts: (i) noise level estimation, mainly solving eigenvector decomposition of subsequence correlation matrix, requires $O\left(L^{3}\right)$ operations; (ii) the sparse-representation solving, if (13) is solved by OMP, requires $O(\mathrm{KMN})$ operations [24].

\section{Results and Discussion}

In this section, both simulated experiments and real data are explored to verify the denoising performance of SRDN-NS.

4.1. Simulated Experiments Setup. We simulate the noisy radar return of a target with 5 scatterers to verify the effectiveness of SRDN-NS. The scatterer distribution and radar system parameters are shown in Tables 1 and 2, respectively.

Under the above system parameters, every sampling sequence of a single pulse is a 256-length vector. Assuming the reference range for dechirping is $12010 \mathrm{~m}$, the noiseless HRRP obtained by FFT is illustrated in Figure 1.

The number of scatterers is 5, much less than the 256 range cells. Thus the sampling sequence is a sparse vector 
TABLE 1: Scatterer distribution.

\begin{tabular}{lcc}
\hline Scatterer & Range $(\mathrm{m})$ & Amplitude of return \\
\hline 1 & 12000 & 0.8 \\
2 & 12002.1 & 0.5 \\
3 & 12005.3 & 0.4 \\
4 & 12006.7 & 1 \\
5 & 12010 & 0.7 \\
\hline
\end{tabular}

TABLE 2: Radar system parameters.

\begin{tabular}{lc}
\hline Parameter name & Value \\
\hline Carrier frequency & $5.52 \mathrm{GHz}$ \\
Bandwidth & $400 \mathrm{MHz}$ \\
Pulse width & $25 \mathrm{us}$ \\
Reference pulse width & $25.6 \mathrm{us}$ \\
Sampling rate for dechirped return & $10 \mathrm{MHz}$ \\
\hline
\end{tabular}

in frequency domain. To produce noisy return, we add the complex Gaussian white noise (CGWN) into the noise-free return with SNR defined as

$$
\mathrm{SNR}_{\text {Orig }}=\frac{\sum_{k=1}^{K} A_{k}^{2}}{\sigma^{2}},
$$

where $A_{k}$ is the amplitude of the return from $k$ th scatterer, $\sigma^{2}$ is the variance of CGWN, and $K$ denotes the number of scatterers.

4.2. Noise Level Estimation. Noise level estimation is a critical problem for SRDN-NS. In the first experiment, we surveyed the estimation accuracy of $\delta$ as discussed in Section 3.2. The length of sliding window is 60 . As a comparison, the selecting noise cells method [12] and wavelet method [13] are also utilized to estimate the noise level. In wavelet method, the "db8" wavelet basis is chosen for its experimental superiority over other "db" wavelet bases. The dechirped return is decomposed into 8 layers of wavelet. To verify the estimation performance of these methods, the absolute estimation error $|\widehat{\delta}-\delta|$, where $\widehat{\delta}$ is the estimation value of noise level $\delta$, is investigated by a Monte Carlo experiment. 100 trails are performed at every SNR, and the results with SNR varying from $-10 \mathrm{~dB}$ to $20 \mathrm{~dB}$ are shown in Figure 2. It can be seen that, in general, the proposed sliding subsequence method has better estimation performance than selecting noise cells and wavelet. Notice that the estimation error of selecting noise cells method is not stable reducing with SNR increasing. That is because, with SNR increasing, the sidelobe of HRRP introduces much error; besides the threshold which determines noise cells is not easy to select.

4.3. Denoising Performance. In this subsection, we investigate the denoising performance of SRDN-NS. The redundant dictionary is established according to (10) with $M=256$ and $N=1024,2048$, respectively, for different experiments. OMP is employed to solve (13) for recovering the denoised return. For comparing denoising performance, SRDN with noise

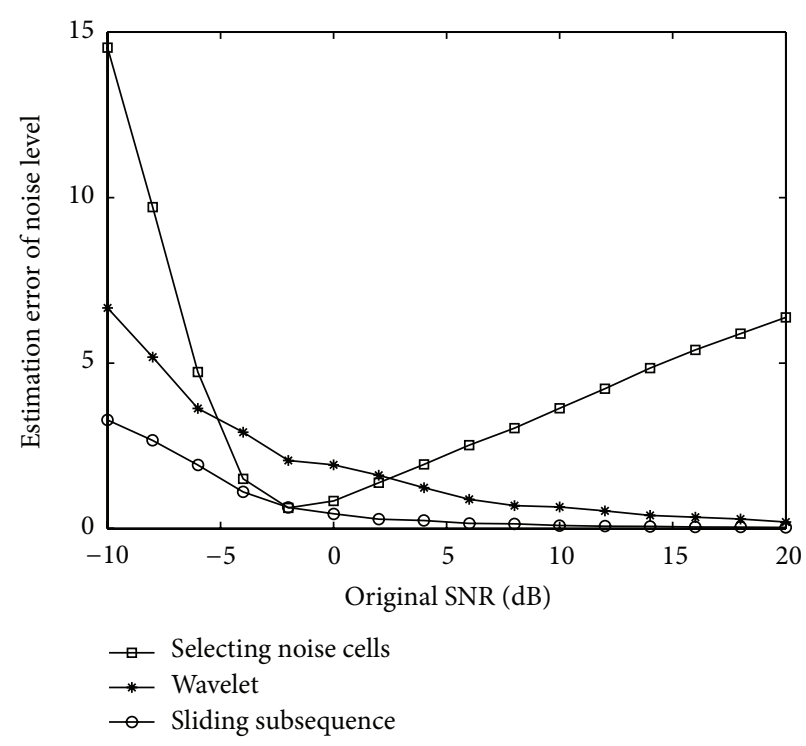

FIGURE 2: Estimation error of noise level by various methods with SNR varying from $-10 \mathrm{~dB}$ to $20 \mathrm{~dB}$.

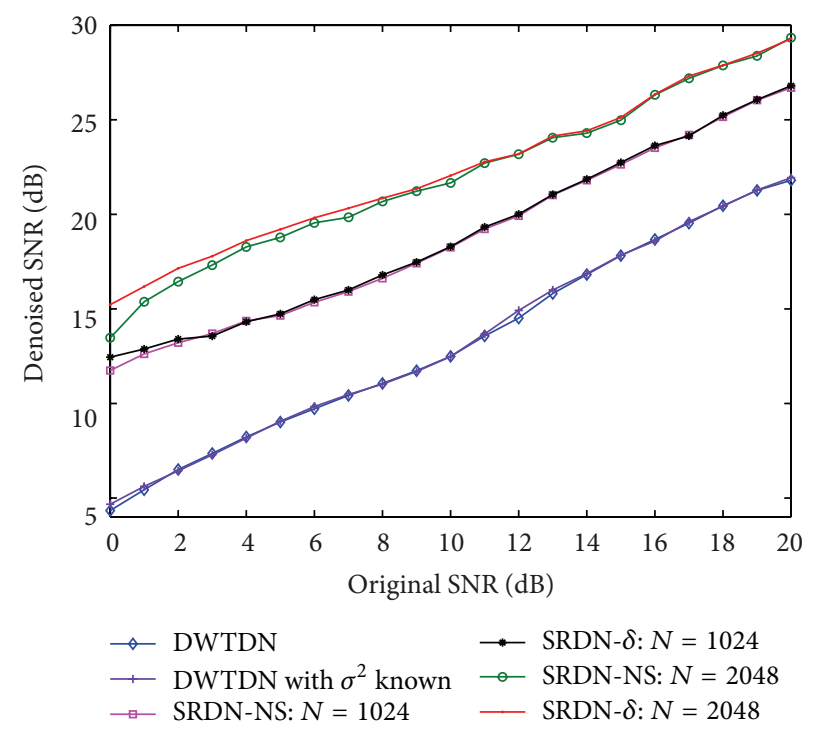

FIGURE 3: SNR of denoised return by various methods with original SNR varying from $0 \mathrm{~dB}$ to $20 \mathrm{~dB}$.

level known (referring to it as $\mathrm{SRDN}-\delta$ ) and the traditional DWTDN as discussed in $[14,28,29]$ are also utilized to reduce noise in the return. In DWTDN, "db8" wavelet basis is chosen and the dechirped return is decomposed into 8 layers of wavelet, and then the Heursure threshold is used to reject noise. For full comparison, DWTDN with noise variance known is also presented, and the soft threshold is selected as $\lambda=\sigma \sqrt{2 \ln M}$.

To verify the denoising performance, we define SNR of the denoised return as

$$
\mathrm{SNR}_{d}=10 \log _{10} \frac{\|\mathbf{s}\|_{2}^{2}}{\|\widehat{\mathbf{s}}-\mathbf{s}\|_{2}^{2}},
$$



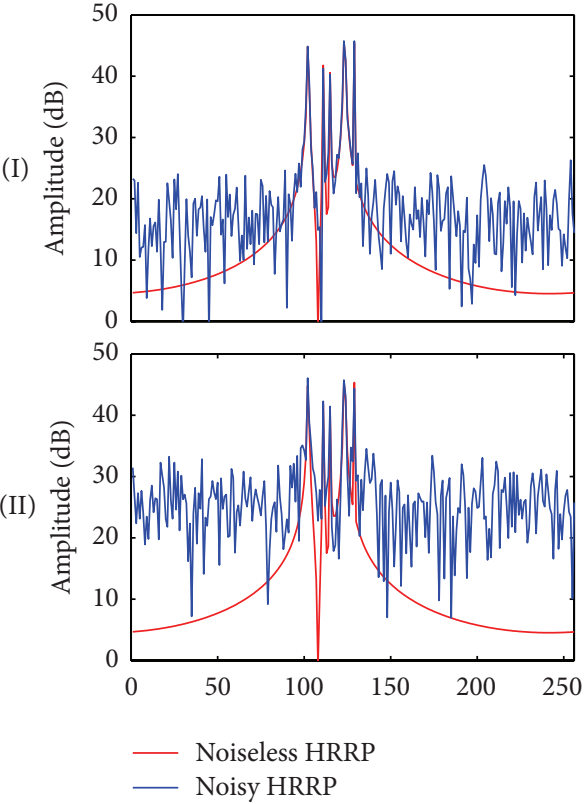

(a)
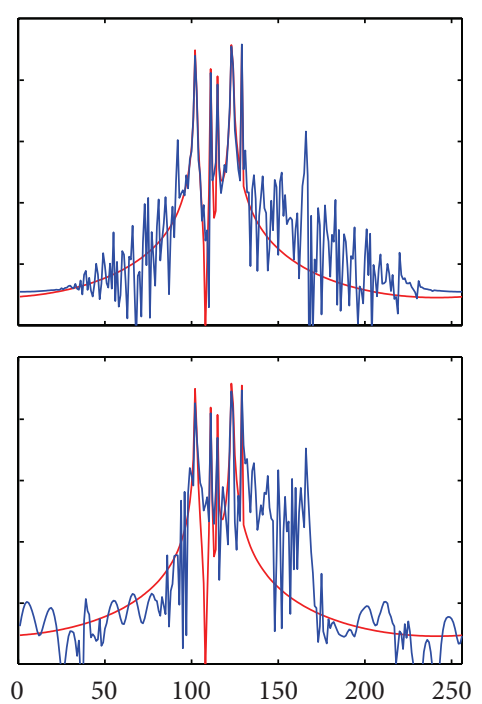

— Noiseless HRRP
HRRP with DWTDN

(b)
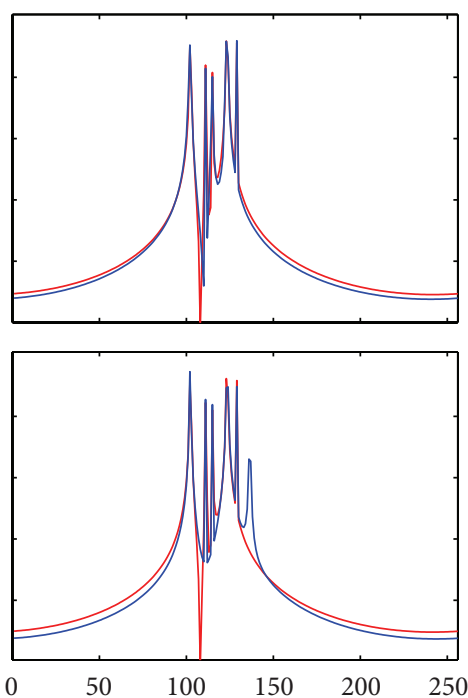

- Noiseless HRRP

— HRRP with SRDN-NS

(c)

FIGURE 4: Profiling results of simulated return: (a) noiseless HRRP and noisy HRRP at $10 \mathrm{~dB}$ (I) and noisy HRRP at $1 \mathrm{~dB}$ (II); (b) noiseless HRRP and HRRP with DWTDN; (c) noiseless HRRP and HRRP with SRDN-NS.

where $\mathbf{s}$ denotes the original noise-free return and $\widehat{\mathbf{s}}$ denotes the denoised return. $\|\widehat{\mathbf{s}}-\mathbf{s}\|_{2}^{2}$ stands for the noise components and information loss in the denoised return. 100 Monte Carlo trails are performed to compute $\mathrm{SNR}_{d}$ at every SNR. The denoising results of these methods with original SNR varying from $0 \mathrm{~dB}$ to $20 \mathrm{~dB}$ are showed in Figure 3.

It can be seen from Figure 3 that SRDN presents much better denoising performance than DWTDN. This is because the dechirped radar return is composed of complex sinusoidal components. It is more sparsely represented in the Fourier redundant dictionary than in wavelet basis; it follows that the signal energy is more concentrated in the Fourier redundant dictionary, thus leading to a better denoising performance. Note that the denoising performance of SRDN improves with $N$ increasing. The reason is that, with $N$ increasing, A gradually improves frequency resolution, and the dechirped signal can be represented more exactly with less atoms in A. But $N$ cannot increase infinitely because of the limitation of computational cost. In addition, compared with $\mathrm{SRDN}-\delta$, SRDN-NS provides a close performance; in other words, the denoising performance of SRDN-NS is approaching the performance upper bound of SRDN.

4.4. Profiling Results. In this subsection, the profiling results by FFT after SRND-NS denoising are illustrated in Figure 4. The experimental parameters are the same as the experiment in Section 4.3. The original noisy HRRPs at different SNRs are shown in Figure 4(a), $10 \mathrm{~dB}$ in (I) and $1 \mathrm{~dB}$ in (II). For comparison, the profiling results with DWTDN are presented in Figure 4(b), which still contain some noise components around signal part, whereas SRDN-NS provides precise and clear HRRPs in Figure 4(c). Notice that, in low SNR scenario, the denoised return by SRDN-NS may lose some weakened signal components or still contain a little strong noise (as shown in (II) of Figure 4(c)) due to the estimation error of noise level.

4.5. Real Data Profiling Results. We apply SRDN-NS to denoise the real return of $\mathrm{AN}-26$ plane under different noise levels in this subsection. This dataset was previously described and utilized in [10]. The radar system parameters are the same as the simulated experimental parameters in Table 2. The profiling results by FFT after denoising by DWTDN and SRDN-NS are shown in Figure 5. The noisy HRRPs with different noise levels are shown in Figure 5(a), high SNR (I) and low SNR (II). Figure 5(b) shows that the HRRPs with DWTDN still contain some noise components around the signal part, whereas, as shown in Figure 5(c), SRDN-NS provides much clearer HRRPs and preserves most of the information.

\section{Conclusions}

This work has developed an effective denoising method, SRDN-NS, for high resolution range profiling at low SNR. The denoised return is recovered by solving a sparse representation problem. Simulation and real data results demonstrate that SRDN-NS can greatly enhance SNR of radar return, leading to a high-quality HRRP. In this work, we only consider the influence of the zero-mean Gaussian white noise; however, there are many other complicated noise environments, such as ground clutter and sea clutter, that the radar system may 


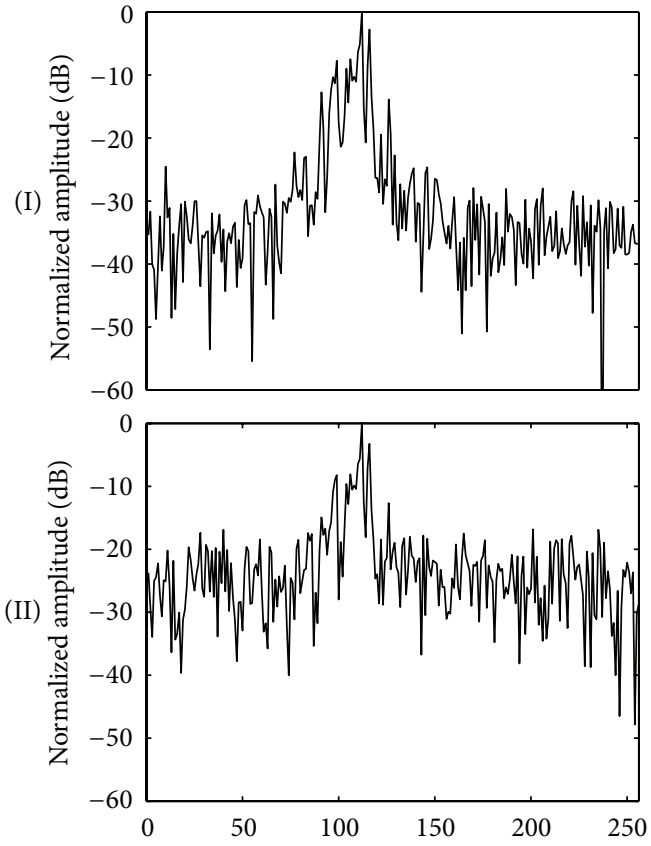

(a)
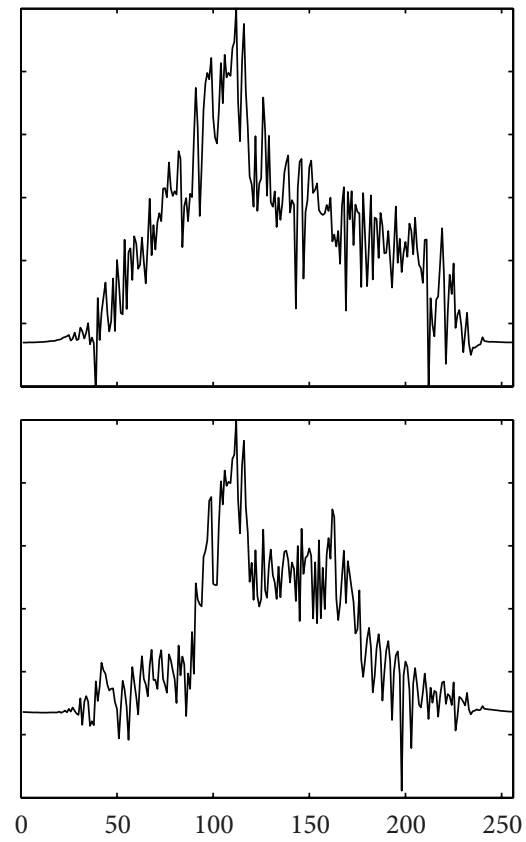

(b)
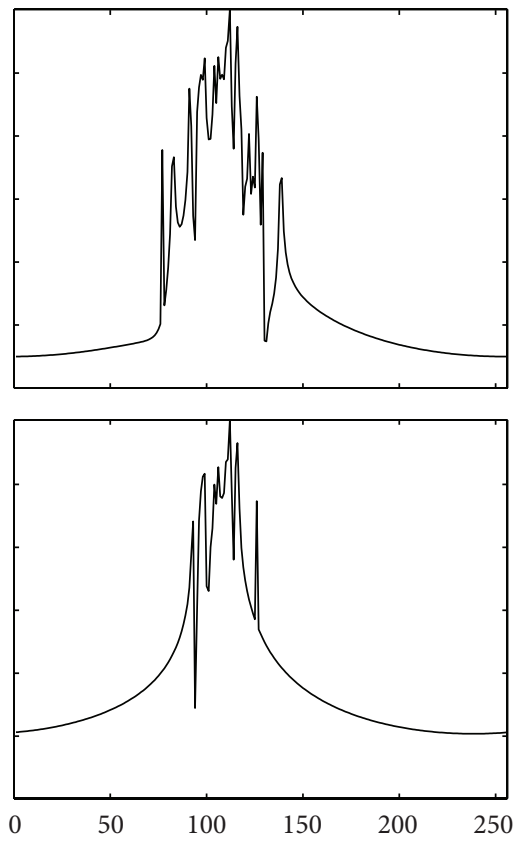

(c)

FIGURE 5: Profiling results of AN-26 real return: (a) the noisy HRRPs at high SNR (I) and low SNR (II), respectively; (b) HRRPs with DWTDN; (c) HRRPs with SRDN-NS. All HRRPs are normalized by maximum component absolute mode and on the same scale.

face, so in the future we will continue our study on these complicated environments.

\section{Conflict of Interests}

The authors declare that there is no conflict of interests regarding the publication of this paper.

\section{Acknowledgment}

This work was supported by the National Science Foundation of China under Grant no. 61201311.

\section{References}

[1] S. K. Wong, "High range resolution profiles as motion-invariant features for moving ground targets identification in SAR-based automatic target recognition," IEEE Transactions on Aerospace and Electronic Systems, vol. 45, no. 3, pp. 1017-1039, 2009.

[2] J. Gudnason, J. Cui, and M. Brookes, "HRR automatic target recognition from superresolution scattering center features," IEEE Transactions on Aerospace and Electronic Systems, vol. 45, no. 4, pp. 1512-1524, 2009.

[3] K. Copsey and A. Webb, "Bayesian gamma mixture model approach to radar target recognition," IEEE Transactions on Aerospace and Electronic Systems, vol. 39, no. 4, pp. 1201-1217, 2003.

[4] L. Du, H. Liu, and Z. Bao, "Radar HRRP statistical recognition based on hypersphere model," Signal Processing, vol. 88, no. 5, pp. 1176-1190, 2008.
[5] L. Du, P. Wang, H. Liu, M. Pan, F. Chen, and Z. Bao, "Bayesian spatiotemporal multitask learning for radar HRRP target recognition," IEEE Transactions on Signal Processing, vol. 59, no. 7, pp. 3182-3196, 2011.

[6] L. Du, H. Liu, Z. Bao, and J. Zhang, "Radar automatic target recognition using complex high-resolution range profiles," IET Radar Sonar \& Navigation, vol. 1, no. 1, pp. 18-26, 2007.

[7] L. Du, H. Liu, Z. Bao, and M. Xing, "Radar HRRP target recognition based on higher order spectra," IEEE Transactions on Signal Processing, vol. 53, no. 7, pp. 2359-2368, 2005.

[8] R. O. Duda, P. E. Hart, and D. G. Stork, Pattern Classification, John Wiley \& Sons, New York, NY, USA, 2nd edition, 2012.

[9] I. Jouny, E. D. Garber, and R. L. Moses, "Radar target identification using the bispectrum: a comparative study," IEEE Transactions on Aerospace and Electronic Systems, vol. 31, no. 1, pp. 69-77, 1995.

[10] L. Du, H. W. Liu, P. H. Wang, B. Feng, M. Pan, and Z. Bao, "Noise robust radar HRRP target recognition based on multitask factor analysis with small training data size," IEEE Transactions on Signal Processing, vol. 60, pp. 3546-3559, 2012.

[11] Z. She and Y. Liu, "Autofocus for ISAR imaging using higher order statistics," IEEE Geoscience and Remote Sensing Letters, vol. 5, no. 2, pp. 299-303, 2008.

[12] L. Zhang, M. Xing, C.-W. Qiu et al., "Resolution enhancement for inversed synthetic aperture radar imaging under low SNR via improved compressive sensing," IEEE Transactions on Geoscience and Remote Sensing, vol. 48, no. 10, pp. 3824-3838, 2010.

[13] G. Xu, M. Xing, L. Zhang, Y. Liu, and Y. Li, "Bayesian inverse synthetic aperture radar imaging," IEEE Geoscience and Remote Sensing Letters, vol. 8, no. 6, pp. 1150-1154, 2011.

[14] B. M. Huether, S. C. Gustafson, and R. P. Broussard, "Wavelet preprocessing for high range resolution radar classification," 
IEEE Transactions on Aerospace and Electronic Systems, vol. 37, no. 4, pp. 1321-1332, 2001.

[15] A. Achim, P. Tsakalides, and A. Bezerianos, "SAR image denoising via Bayesian wavelet shrinkage based on heavy-tailed modeling," IEEE Transactions on Geoscience and Remote Sensing, vol. 41, no. 8, pp. 1773-1784, 2003.

[16] A. V. Totsky, I. V. Kurbatov, V. V. Lukin, K. O. Egiazarian, and J. T. Astola, "Combined bispectrum-filtering techniques for radar output signal reconstruction in ATR applications," in Automatic Target Recognition XIII, vol. 5094 of Proceedings of SPIE, pp. 301-312, April 2003.

[17] V. Lukin, A. Totsky, D. Fevralev, A. Roenko, J. Astola, and K. Egiazarian, "Adaptive combined bispectrum-filtering signal processing in radar systems with low SNR," in Proceedings of the IEEE International Symposium on Circuits and Systems (ISCAS '06), pp. 3690-3693, May 2006.

[18] L. Du, H. Liu, Z. Bao, and J. Zhang, "A two-distribution compounded statistical model for radar HRRP target recognition," IEEE Transactions on Signal Processing, vol. 54, no. 6, pp. 22262238, 2006.

[19] S. S. Chen, D. L. Donoho, and M. A. Saunders, "Atomic decomposition by basis pursuit," SIAM Review, vol. 43, no. 1, pp. 129159, 2001.

[20] D. L. Donoho, M. Elad, and V. N. Temlyakov, "Stable recovery of sparse overcomplete representations in the presence of noise," IEEE Transactions on Information Theory, vol. 52, no. 1, pp. 6-18, 2006.

[21] M. Babaie-Zadeh and C. Jutten, "On the stable recovery of the sparsest overcomplete representations in presence of noise," IEEE Transactions on Signal Processing, vol. 58, pp. 5396-5400, 2010.

[22] D. R. Wehner, High Resolution Radar, vol. 1, Artech House, Norwood, Mass, USA, 1987.

[23] W. J. Caputi, "Stretch: a time-transformation technique," IEEE Transactions on Aerospace and Electronic Systems, vol. 7, no. 2, pp. 269-278, 1971.

[24] J. A. Tropp and A. C. Gilbert, "Signal recovery from random measurements via orthogonal matching pursuit," IEEE Transactions on Information Theory, vol. 53, no. 12, pp. 4655-4666, 2007.

[25] M. Wax and T. Kailath, "Detection of signals by information theoretic criteria," IEEE Transactions on Acoustics, Speech, and Signal Processing, vol. 33, no. 2, pp. 387-392, 1985.

[26] E. Fishler, M. Grosmann, and H. Messer, "Detection of signals by information theoretic criteria: general asymptotic performance analysis," IEEE Transactions on Signal Processing, vol. 50, no. 5, pp. 1027-1036, 2002.

[27] D. Ramakrishna, N. B. Mandayam, and R. D. Yates, "Subspacebased SIR estimation for CDMA cellular systems," IEEE Transactions on Vehicular Technology, vol. 49, no. 5, pp. 1732-1742, 2000.

[28] M. Jansen, Noise Reduction by Wavelet Thresholding, vol. 161, Springer, New York, NY, USA, 2001.

[29] D. L. Donoho, "De-noising by soft-thresholding," IEEE Transactions on Information Theory, vol. 41, no. 3, pp. 613-627, 1995. 

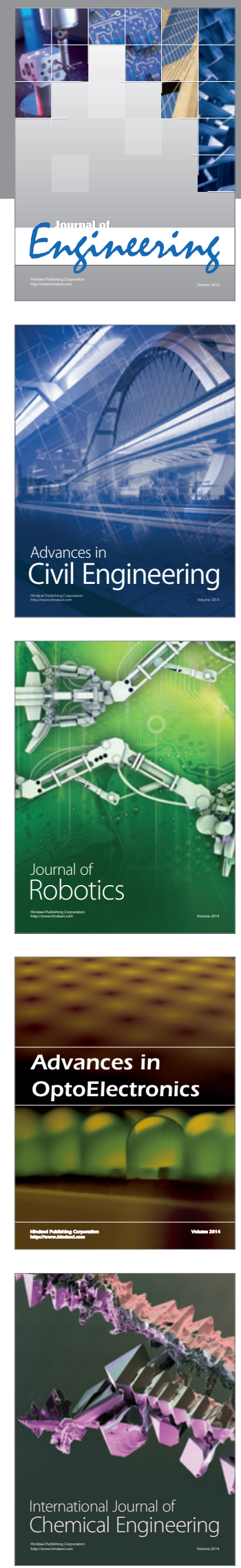

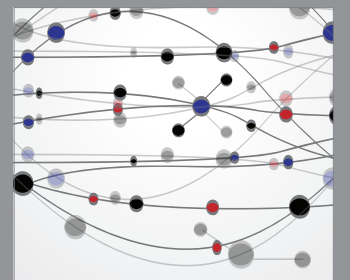

The Scientific World Journal
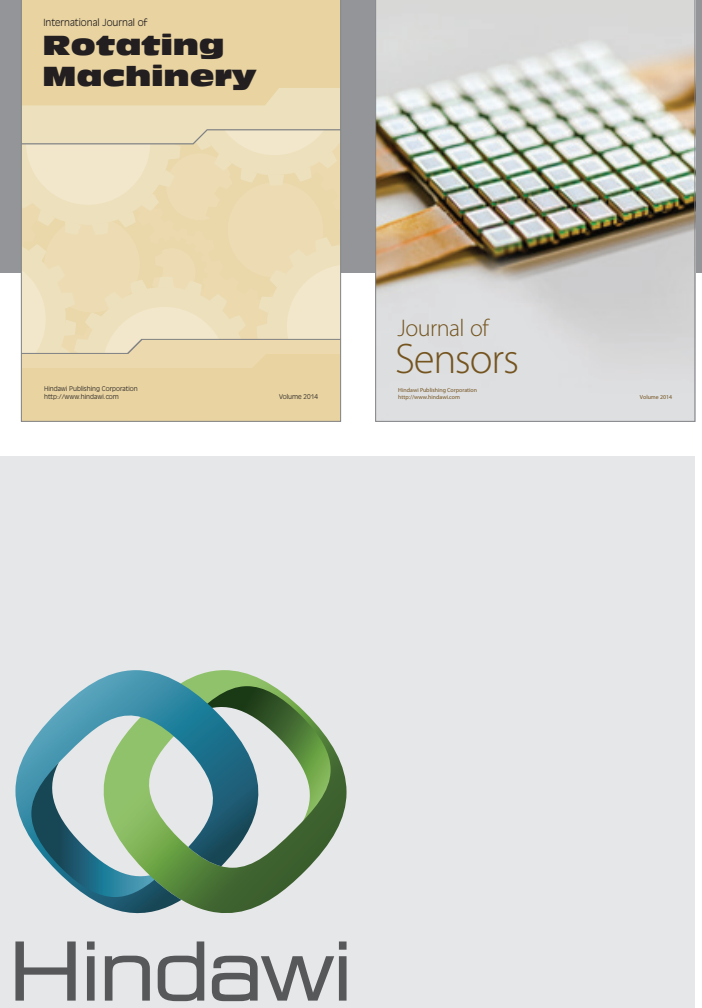

Submit your manuscripts at http://www.hindawi.com
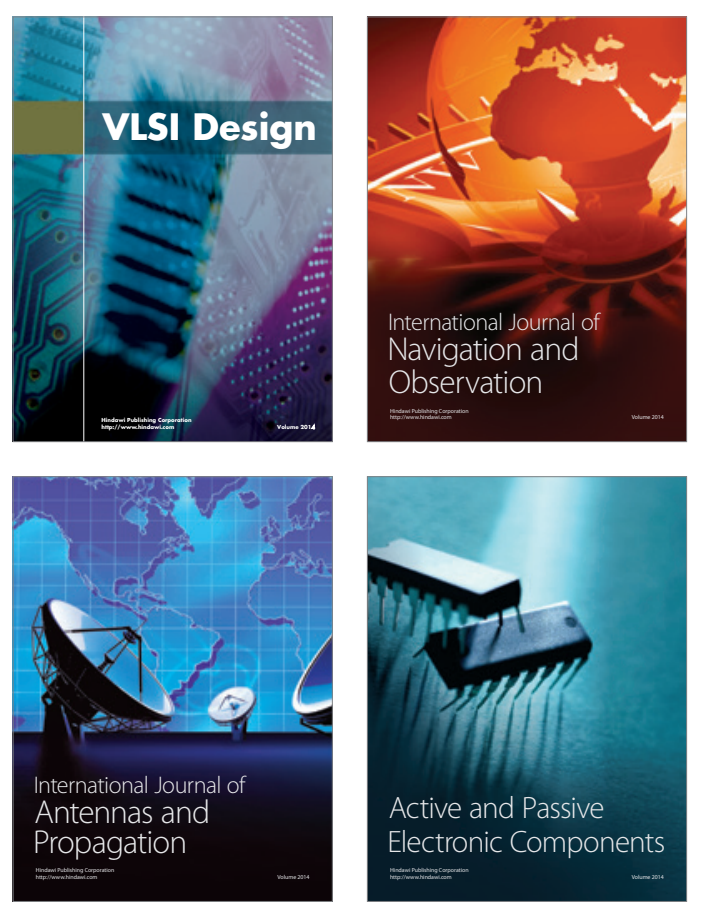
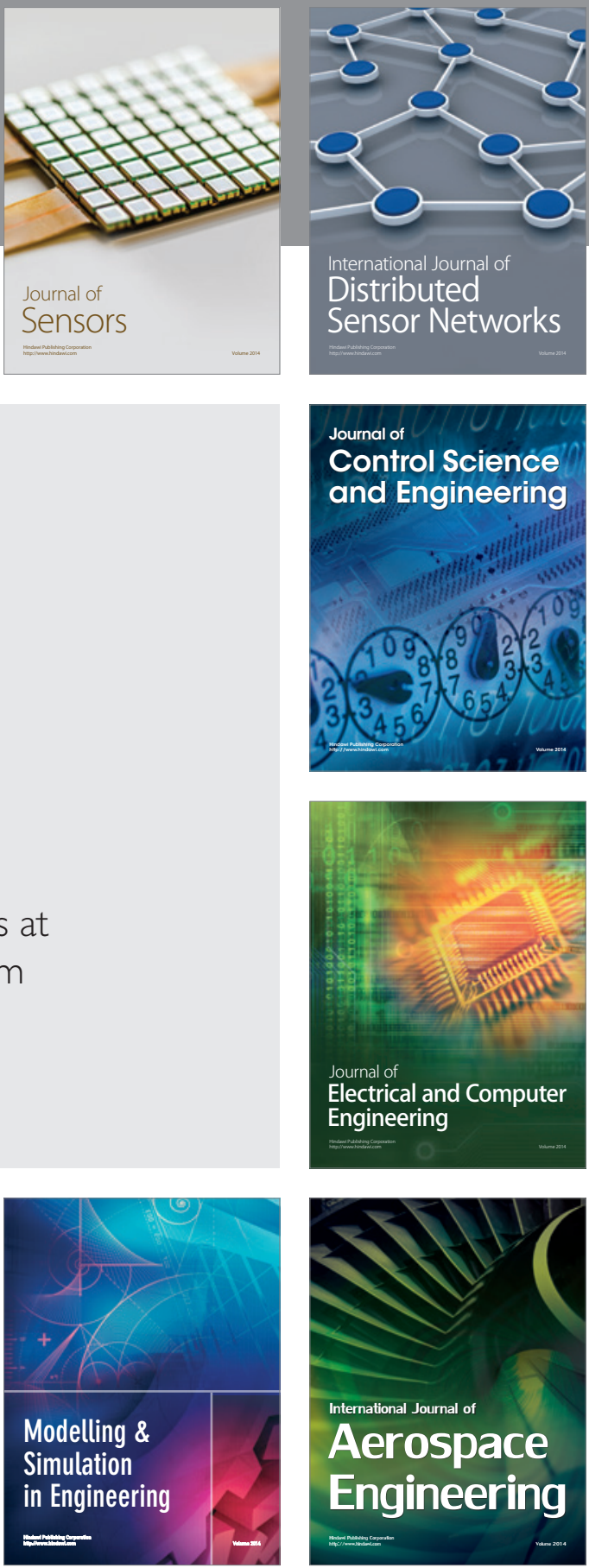

Journal of

Control Science

and Engineering
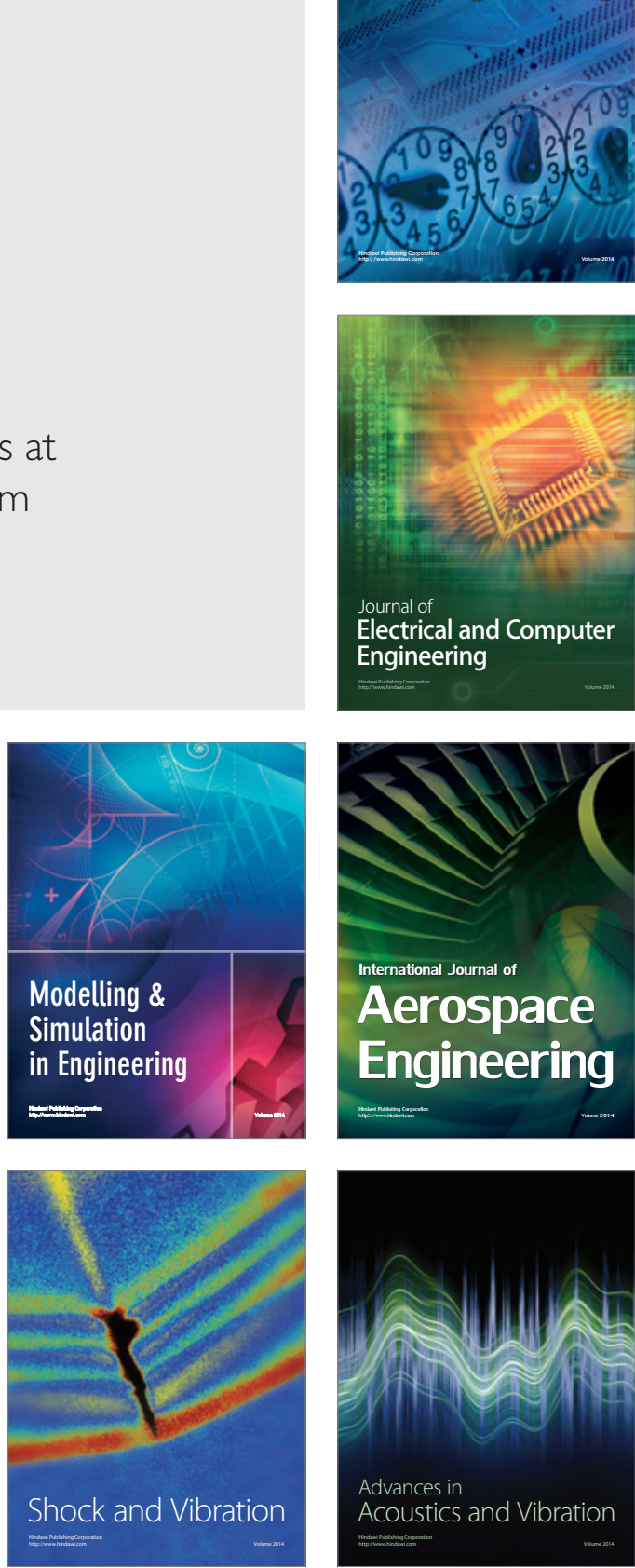